\title{
Analysis of longitudinal head impact exposure and white matter integrity in returning youth football players
}

\author{
Mireille E. Kelley, PhD, ${ }^{1,2}$ Jillian E. Urban, PhD, MPH, ${ }^{1,2}$ Derek A. Jones, PhD, ${ }^{1,2}$ \\ Elizabeth M. Davenport, PhD, ${ }^{3}$ Logan E. Miller, PhD, ${ }^{1,2}$ Beverly M. Snively, PhD, ${ }^{4}$ \\ Alexander K. Powers, MD, ${ }^{5}$ Christopher T. Whitlow, MD, PhD, MHA, ${ }^{1,6,7}$ Joseph A. Maldjian, MD, ${ }^{3}$ \\ and Joel D. Stitzel, PhD ${ }^{1,2}$
}

\begin{abstract}
Departments of ${ }^{1}$ Biomedical Engineering, ${ }^{4}$ Biostatistical Sciences, ${ }^{5}$ Neurosurgery, and ${ }^{6}$ Radiology (Neuroradiology), and ${ }^{7}$ Clinical and Translational Sciences Institute, Wake Forest School of Medicine, Winston-Salem; ${ }^{2}$ Virginia Tech-Wake Forest School of Biomedical Engineering and Sciences, Winston-Salem, North Carolina; and ${ }^{3}$ Department of Radiology, University of Texas Southwestern, Dallas, Texas
\end{abstract}

\begin{abstract}
OBJECTIVE The objective of this study was to characterize changes in head impact exposure (HIE) across multiple football seasons and to determine whether changes in HIE correlate with changes in imaging metrics in youth football players.
\end{abstract}
METHODS On-field head impact data and pre- and postseason imaging data, including those produced by diffusion tensor imaging (DTI), were collected from youth football athletes with at least two consecutive seasons of data. ANCOVA was used to evaluate HIE variations (number of impacts, peak linear and rotational accelerations, and risk-weighted cumulative exposure) by season number. DTI scalar metrics, including fractional anisotropy, mean diffusivity, and linear, planar, and spherical anisotropy coefficients, were evaluated. A control group was used to determine the number of abnormal white matter voxels, which were defined as 2 standard deviations above or below the control group mean. The difference in the number of abnormal voxels between consecutive seasons was computed for each scalar metric and athlete. Linear regression analyses were performed to evaluate relationships between changes in HIE metrics and changes in DTI scalar metrics.

RESULTS There were 47 athletes with multiple consecutive seasons of HIE, and corresponding imaging data were available in a subsample $(n=19)$ of these. Increases and decreases in HIE metrics were observed among individual athletes from one season to the next, and no significant differences (all $p>0.05$ ) in HIE metrics were observed by season number. Changes in the number of practice impacts, 50 th percentile impacts per practice session, and 50 th percentile impacts per session were significantly positively correlated with changes in abnormal voxels for all DTI metrics.

CONCLUSIONS These results demonstrate a significant positive association between changes in HIE metrics and changes in the numbers of abnormal voxels between consecutive seasons of youth football. Reducing the number and frequency of head impacts, especially during practice sessions, may decrease the number of abnormal imaging findings from one season to the next in youth football.

https://thejns.org/doi/abs/10.3171/2021.1.PEDS20586

KEYWORDS diffusion tensor imaging; head acceleration; risk-weighted cumulative exposure; biomechanics; head impact frequency; trauma

$\mathrm{S}$ uBCONCUSSIVE head impacts (i.e., impacts that do not result in acute signs or symptoms of concussion) and concussive head impacts have been evaluated in football athletes by using head acceleration measurement devices..$^{1-7}$ Studies have characterized football players' subconcussive head impact exposure (HIE), including the magnitude and frequency of impacts, during a season of play. Overall, there are general trends of increasing HIE with increasing levels of play from youth through college age. ${ }^{1,4}$ There is also considerable variability in HIE among individuals. Youth and high school football players can sustain 26 to 1003 and 129 to 1258 impacts in a season, re-

ABBREVIATIONS $C_{L}=$ linear anisotropy coefficient; $C_{P}=$ planar anisotropy coefficient; $C_{S}=$ spherical anisotropy coefficient; $C T E=$ chronic traumatic encephalopathy; $\mathrm{DTI}=$ diffusion tensor imaging; $\Delta$ = change in; FA = fractional anisotropy; HIE = head impact exposure; HIT = Head Impact Telemetry; MD = mean diffusivity; RWE = riskweighted cumulative exposure; $\mathrm{RWE}_{\mathrm{CP}}=\mathrm{RWE}$, combined probability.

SUBMITTED August 11, 2020. ACCEPTED January 11, 2021.

INCLUDE WHEN CITING Published online June 15, 2021; DOI: 10.3171/2021.1.PEDS20586. 
spectively. ${ }^{4,5}$ Factors such as practice drills, season length, and player position can influence $\mathrm{HIE}, 4,8-10$ however, there is a limited understanding of how an individual player's HIE changes over consecutive seasons of participation in football. ${ }^{4,11,12}$

A growing body of evidence has demonstrated that accumulation of repetitive concussive and subconcussive head impacts throughout many years of participation in contact sports may contribute to later-life neurodegenerative diseases. ${ }^{11,13-15}$ Neuropathological studies have identified chronic traumatic encephalopathy (CTE) in some deceased former football players. ${ }^{14,16}$ While participation in collision sports is a common denominator among individuals with this disease, the pathogenesis of CTE and the effect of confounding factors, such as genetics, age of first exposure to repetitive head impacts, number of concussions, and the frequency and severity of accumulated subconcussive impacts, are still largely unknown. ${ }^{11,15,17,18}$ Additionally, there are examples of individuals without a history of concussion but with a history of exposure to repetitive subconcussive impacts who developed CTE. ${ }^{14,19,20}$ This highlights the potential influence of subconcussive impacts on the development of later-life neurological impairments, independent of concussion. Conversely, there are also individuals who have been exposed to repetitive head impacts but do not develop later-life cognitive deficits or other symptoms of CTE. ${ }^{16,21}$

Current research efforts seek to characterize the potential short- and long-term effects of subconcussive head impacts on the brain in contact sport athletes..$^{11,22-26}$ Changes in white matter integrity can be measured using diffusion tensor imaging (DTI) and have been associated with concussion and repetitive subconcussive HIE. ${ }^{25,27,28}$ Studies have demonstrated significant positive associations between subconcussive HIE metrics and changes from pre- to postseason in brain imaging metrics among football players during a single season of play, even in the absence of a clinically diagnosed concussion. ${ }^{22-25,29}$ This is concerning as changes in DTI metrics have also been associated with autism, depression, and migraines in the pediatric population, followed by Alzheimer's disease and other neurodegenerative disorders later in life..$^{30-33}$ Continued research is needed, however, especially in athletes $\leq$ 18 years of age, since athletes in this age group are undergoing structural brain growth and development of memory, problem-solving, and other cognitive skills. ${ }^{34}$

There has been growing concern about the safety of participating in football, especially at the youth level, which may in part be due to increasing awareness of concussion and potential later-life effects of head impacts; however, it has not yet been determined how repetitive head impacts may affect the brain over multiple seasons of participation in youth football..$^{22,24,35}$ Longitudinal data sets covering high school and collegiate athletes have been evaluated to determine whether changes in white matter integrity persist after periods of no-contact rest, but there are conflicting results. . $^{23,36,37}$

Longitudinal biomechanical and imaging data sets are needed to understand how HIE and white matter integrity change over time in individuals. Thus, this study has the following objectives: 1) characterize changes in HIE across football seasons and 2) evaluate whether changes in HIE correlate with changes in imaging metrics over consecutive seasons among youth football players.

\section{Methods}

Head impact data were collected from youth football athletes during a total of 22 team-seasons between 2012 and 2017. Athletes were placed on teams based on the national governing association's age and weight requirements. ${ }^{4,38}$ The study protocol was approved by the Wake Forest University Health Sciences Institutional Review Board, and participant assent and parental consent were properly obtained for participation in the study. Participation in the study was voluntary.

\section{Head Impact Data Collection}

On-field head impact data were collected by instrumenting the Riddell helmets worn by study participants with the Head Impact Telemetry (HIT) System for all preseason, regular season, and playoff practices and games. The HIT System includes an MxEncoder that is installed within the existing padding structure of the helmet to measure the location and magnitude of head impacts. The accelerometers within the MxEncoder are spring-mounted to allow them to remain in contact with the youth football athlete's head throughout the duration of a head impact, ensuring measurement of head acceleration rather than helmet acceleration. The HIT System also includes a sideline data collection field unit that collects head impact data in real time via radio wave transmission. Trained research assistants set up the sideline data collection field unit and monitored head impact data collection at all practices and games. The data collection methodology used in this study, as well as the data processing algorithm and validation of the HIT System, has been previously described in the literature.,39 All sessions were filmed, and the resulting videos were time-synced with the HIT System laptop to verify the time individual athletes were helmeted. Impacts occurring while the players were not wearing helmets (e.g., in the case of a dropped helmet) were excluded from the data set.

\section{Neuroimaging Data Collection}

Study participants completed a pre- and postseason neuroimaging protocol for each season. A group of 16 noncontact sport, control athletes also completed the same neuroimaging protocol, including a baseline scan and a follow-up scan approximately 4 months later. The control athletes were all male, were of comparable age to the football participants, and participated in noncontact sports including swimming, track, and tennis between the baseline and follow-up scanning sessions. Participants and controls with neurological disorders and/or major psychiatric disorders were excluded. Control athletes with a history of concussion or playing contact sports were also excluded. Magnetic resonance imaging (MRI) data were acquired in accordance with the National Institute of Neurological Disorders and Stroke Common Data Elements advanced protocol recommendations by using a 3T Siemens Skyra MRI scanner (Siemens Healthcare). The MRI acquisi- 


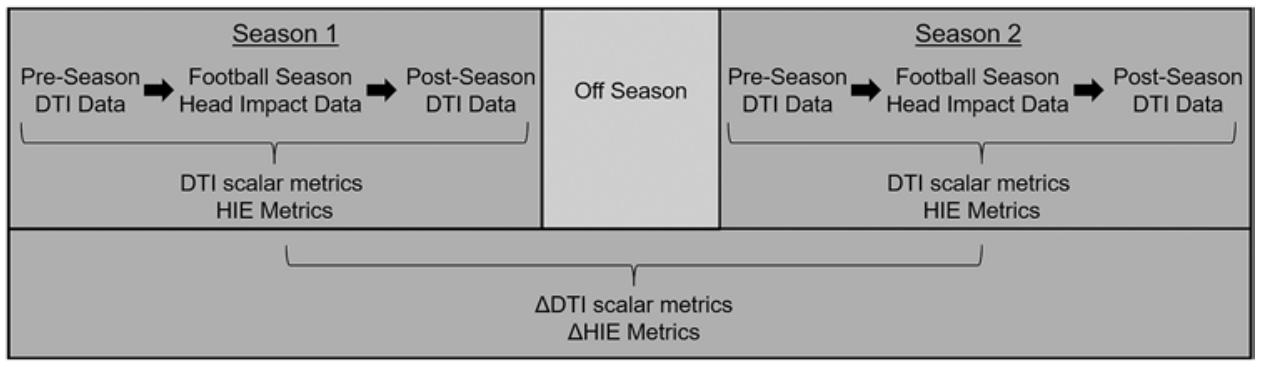

FIG. 1. Summary of HIE and imaging data collection over two consecutive seasons.

tion and processing protocols have been previously published. ${ }^{22,24,25}$ Each scan was reviewed by a radiologist, and youth football athletes and control athletes with clinical abnormalities were excluded from the analysis. Additionally, none of the football players whose data were analyzed received a clinical diagnosis of concussion while enrolled in the study. At the pre- and postseason imaging appointments, additional information, including age, height, and weight, was also collected.

\section{Statistics and Analysis}

Only youth football athletes in whom at least two consecutive seasons of HIE data were available were included in the present analysis. HIE data for each athlete were separated into season numbers (season 1, 2, 3, or 4) based on the number of years the athlete participated in the study. Season 1 indicates the first year of HIE data collection for each athlete but does not indicate the first season the athlete played football, as some athletes had participated on football teams prior to enrolling in the study. HIE was quantified by the number of impacts, peak linear and rotational head accelerations, and risk-weighted cumulative exposure (RWE). The total number of impacts was computed for each athlete-season. From the distribution of the number of impacts per session (i.e., practice and/or game), the 50th percentile was computed for each athlete-season. Also, from the distribution of head accelerations, the 95th percentile linear and rotational accelerations were computed for each athlete-season. The RWE metric combines the magnitude and frequency of head impacts sustained by an athlete on aggregate to describe each athlete's cumulative HIE. 5,40 The risk of concussion associated with each head impact was computed and summed to generate each player's RWE for the season. In this study, logistic regression equations and regression coefficients were used to compute the RWE for each athlete-season by using the combined probability from linear and rotational accelerations $\left(\mathrm{RWE}_{\mathrm{CP}}\right) .{ }^{40}$ Changes in HIE metrics between consecutive seasons were also computed for each athlete (including change in $[\Delta]$ number of impacts, $\Delta 50$ th percentile impacts per session, $\Delta 95$ th percentile linear acceleration $[g], \Delta 95$ th percentile rotational acceleration $\left(\mathrm{rad} / \mathrm{sec}^{2}\right)$, and $\left.\triangle \mathrm{RWE}_{\mathrm{CP}}\right)$. The HIE was evaluated overall for all sessions and stratified by session type (practice or game). For all returning athletes, ANCOVA was used to evaluate differences in HIE among season numbers (seasons 1, 2, and 3) while controlling for repeated measures across seasons.
Team was used as a covariate. For inference, log transformations were applied to the HIE data for ANCOVA due to the right-skewed distribution of the data. Season 4 was excluded from all statistical analyses because a fourth season of data was only available in one participant.

DTI scalar metrics, including fractional anisotropy (FA) and mean diffusivity (MD), as well as shape anisotropy coefficients (linear anisotropy $\left[\mathrm{C}_{\mathrm{L}}\right]$, planar anisotropy $\left[\mathrm{C}_{\mathrm{P}}\right]$, and spherical anisotropy $\left[\mathrm{C}_{\mathrm{S}}\right]$ ), were evaluated in this study. The normative reference for each scalar metric was determined by first computing each control athlete's whole-brain delta (follow-up minus baseline) maps for the DTI scalar metric $\left(\mathrm{FA}, \mathrm{MD}, \mathrm{C}_{\mathrm{L}}, \mathrm{C}_{\mathrm{P}}\right.$, or $\mathrm{C}_{\mathrm{S}}$ ) and then by computing the mean and standard deviation (SD) of the control group's delta maps, providing a normative reference map for each DTI scalar metric. Football athletes' delta maps were computed by subtracting preseason from postseason values for each DTI scalar metric. These delta maps were then compared to the control group's delta maps (mean and SD) to create whole-brain voxel-wise zscore maps for each athlete and each scalar metric. The thresholds for identifying abnormal DTI scalar values in the $\mathrm{z}$-score maps were set at 2 SDs above and below the control group's mean value; therefore, voxels were counted as abnormal voxels if the DTI scalar value either increased or decreased significantly throughout the season compared to control values. A cluster threshold requiring a minimum 1-mL contiguous volume was applied to reduce false positives. For each DTI scalar metric, the total number of abnormal voxels across all white matter and gray matter regions of the brain was recorded for each athlete-season. For between-season comparisons, the difference in the number of abnormal voxels between season 2 and season 1 was computed for each scalar metric and individual athlete for regression analyses $(\triangle \mathrm{DTI}$ scalar metrics: $\Delta \mathrm{FA}_{\text {voxels }}, \Delta \mathrm{MD}_{\text {voxels }}, \Delta \mathrm{C}_{\mathrm{L} \text {-voxels }}, \Delta \mathrm{C}_{\mathrm{P} \text {-voxels }}$, and $\Delta \mathrm{C}_{\mathrm{S} \text { - }}$ voxels). Figure 1 summarizes the HIE and imaging data collection for two consecutive seasons.

Linear regression analyses were performed to evaluate the relationships between changes in HIE metrics and changes in DTI scalar metrics between consecutive seasons of participation in youth football. Due to the small sample size of athletes with 3 complete and consecutive years of imaging and biomechanics data $(\mathrm{N}=6)$, only changes between season 1 and season 2 were evaluated in the regression analyses. Changes in DTI scalar metrics $\left(\Delta \mathrm{DTI}\right.$ scalar metrics: $\Delta \mathrm{FA}_{\text {voxels }}, \Delta \mathrm{MD}_{\text {voxels }}, \Delta \mathrm{C}_{\mathrm{L} \text {-voxels }}, \Delta \mathrm{C}_{\mathrm{P}-}$ voxels , and $\left.\Delta \mathrm{C}_{\mathrm{S} \text {-voxels }}\right)$ were used as the dependent variables. 
TABLE 1. Summary of youth football athletes' age, height, and weight for all 109 athlete-seasons

\begin{tabular}{|c|c|c|c|c|c|}
\hline & Season $1(N=47)$ & Season $2(N=47)$ & Season $3(N=14)$ & Season $4(N=1)$ & All Seasons $(N=109)$ \\
\hline Age in yrs & $11.7(10.6-12.0)$ & $12.7(11.6-13.0)$ & $12.7(12.3-13.1)$ & 13.6 & $12.1(11.4-12.8)$ \\
\hline Height in inches* & $59.6(57.8-62.5)$ & $63.5(61.3-65.4)$ & $64.5(61.9-66.9)$ & 68.3 & $62.1(59.4-64.8)$ \\
\hline Weight in Ibs† & $104.7(92.7-115.7)$ & $118.6(108.2-134.4)$ & $129.8(114.8-141.4)$ & 141.0 & $112.8(101.2-130.0)$ \\
\hline
\end{tabular}

Changes in HIE measures ( $\Delta$ number of impacts, $\Delta 50$ th percentile impacts per session, $\Delta 95$ th percentile linear acceleration $[g], \Delta 95$ th percentile rotational acceleration $\left(\mathrm{rad} / \mathrm{s}^{2}\right)$, and $\left.\triangle \mathrm{RWE} \mathrm{EP}_{\mathrm{CP}}\right)$ were used as independent variables. The linear models were also adjusted for the covariates of the average age of the athletes between season 1 and season 2 preseason scans and the difference in pre- to postseason scan times between the two seasons. Partial $\mathrm{R}^{2}$ values were used to describe the strength of the associations after accounting for systematic differences among ages and differences in time between scans. All statistical analyses were performed using SAS software version 9.4 (SAS Institute Inc.).

\section{Results}

On-field head impact data were collected from 195 athletes, spanning 6 years and totaling 259 athlete-seasons. Athletes for whom only a single season of head impact data was available were excluded from this analysis, resulting in inclusion in this study of 47 individual youth football athletes with multiple consecutive years of head impact data (Table 1). Among the group of returning athletes, 2 years of head impact data were available in 33 athletes, 3 years of data were available in 13 athletes, and 4 years of data were available in only 1 athlete. This resulted in 109 athlete-seasons of HIE data among the 47 individual returning youth football athletes.

Data on a total of 41,148 head impacts were collected across the 109 athlete-seasons. The median (95th percentile) linear and rotational accelerations of all impacts were $20.4(52.5) \mathrm{g}$ and $973.6(2458.7) \mathrm{rad} / \mathrm{sec}^{2}$, respectively. The average number of practices and games during which an athlete received at least one impact per season were $24 \pm 7$ and $9 \pm 2$, respectively.

Changes in HIE measures varied among returning athletes, with some athletes experiencing increases in HIE and other athletes experiencing decreases in HIE from one season to the next (Fig. 2). As seen in Table 2, there were trends of increasing mean number of game impacts, mean 50th percentile impacts per session, and mean 50th percentile impacts per game from season 1 through season 3 within the sample, but the differences between seasons were not significant. Overall, among all returning athletes, there were no significant differences (all p > 0.05) in HIE metrics among season numbers (i.e., seasons 1, 2, and 3). Nevertheless, it should be noted that there was significant variability in several HIE metrics among teams, as mentioned in Table 2.

Consecutive seasons of pre- and postseason MRI data were acquired for 23 of the 47 individual returning youth football athletes; however, 4 of these athletes were excluded due to neurological disorders or clinical abnormalities found in their imaging studies. This resulted in 19 athletes for whom complete imaging and HIE data for two consecutive seasons were included in the regression analyses. MR images identifying changes in abnormal voxels from one season to the next in 2 football athletes are shown in Fig. 3. The median (IQR) percent changes in the number of abnormal voxels between seasons for each DTI scalar metric were as follows: $\Delta \mathrm{FA}_{\text {voxels }},-6.6 \%(-62.0 \%$ to $122.6 \%) ; \Delta \mathrm{MD}_{\text {voxels }}, 63.3 \%$ ( $-39.9 \%$ to $\left.234.1 \%\right) ; \Delta \mathrm{C}_{\mathrm{L} \text {-voxels }}$, $-20.6 \%$ ( $-57.9 \%$ to $110.8 \%) ; \Delta \mathrm{C}_{\text {P-voxels }},-26.1 \%(-39.7 \%$ to $40.8 \%)$; and $\Delta \mathrm{C}_{\mathrm{S} \text {-voxels }},-21.8 \%$ (-55.1\% to $\left.157.1 \%\right)$. Positive percent changes indicate a higher number of abnormal voxels in season 2 than in season 1 , and negative percent changes indicate a lower number of abnormal voxels in season 2 than in season 1. Both increases and decreases in the number of abnormal voxels between season 1 and season 2 were observed among individual athletes.

As seen in Table 3, the change in the total number of practice impacts from season 1 to season 2 demonstrated a significant positive association with all $\triangle D T I$ scalar metrics. Changes in the 50th percentile number of impacts per session and the 50th percentile number of impacts per practice were also significantly positively correlated with $\Delta \mathrm{FA}_{\text {voxels }}, \Delta \mathrm{C}_{\mathrm{L} \text {-voxels }}, \Delta \mathrm{C}_{\mathrm{P} \text {-voxels }}$, and $\Delta \mathrm{C}_{\mathrm{S} \text {-voxels }}$ (Fig. 3). Examples of the positive linear relationship between the change in the number and frequency of practice impacts and $\triangle$ DTI scalar metrics are shown in Fig. 4. There was a trend toward statistical significance $(p<0.1)$ for a positive association between change in the total number of impacts per season and all $\triangle D T I$ scalar metrics. Changes in the $\mathrm{RWE}_{\mathrm{CP}}$ and the 95th percentile linear and rotational accelerations were not significantly associated with any $\triangle \mathrm{DTI}$ scalar metrics.

\section{Discussion}

The results from this study suggest that, among individual athletes, the number of abnormal DTI voxels can increase or decrease between football seasons, and that neuroimaging changes across consecutive seasons are related to changes in some of the HIE metrics examined. Specifically, the $\Delta$ number of practice impacts was significantly positively associated with all $\Delta$ DTI scalar metrics. Also, the $\Delta 50$ th percentile impacts per practice and the $\Delta 50$ th percentile impacts per session were significantly positively correlated with $\Delta \mathrm{FA}_{\text {voxels }}, \Delta \mathrm{C}_{\mathrm{L} \text {-voxels }}, \Delta \mathrm{C}_{\mathrm{P} \text {-voxels }}$, and $\Delta \mathrm{C}_{\mathrm{S} \text {-voxels }}$. 

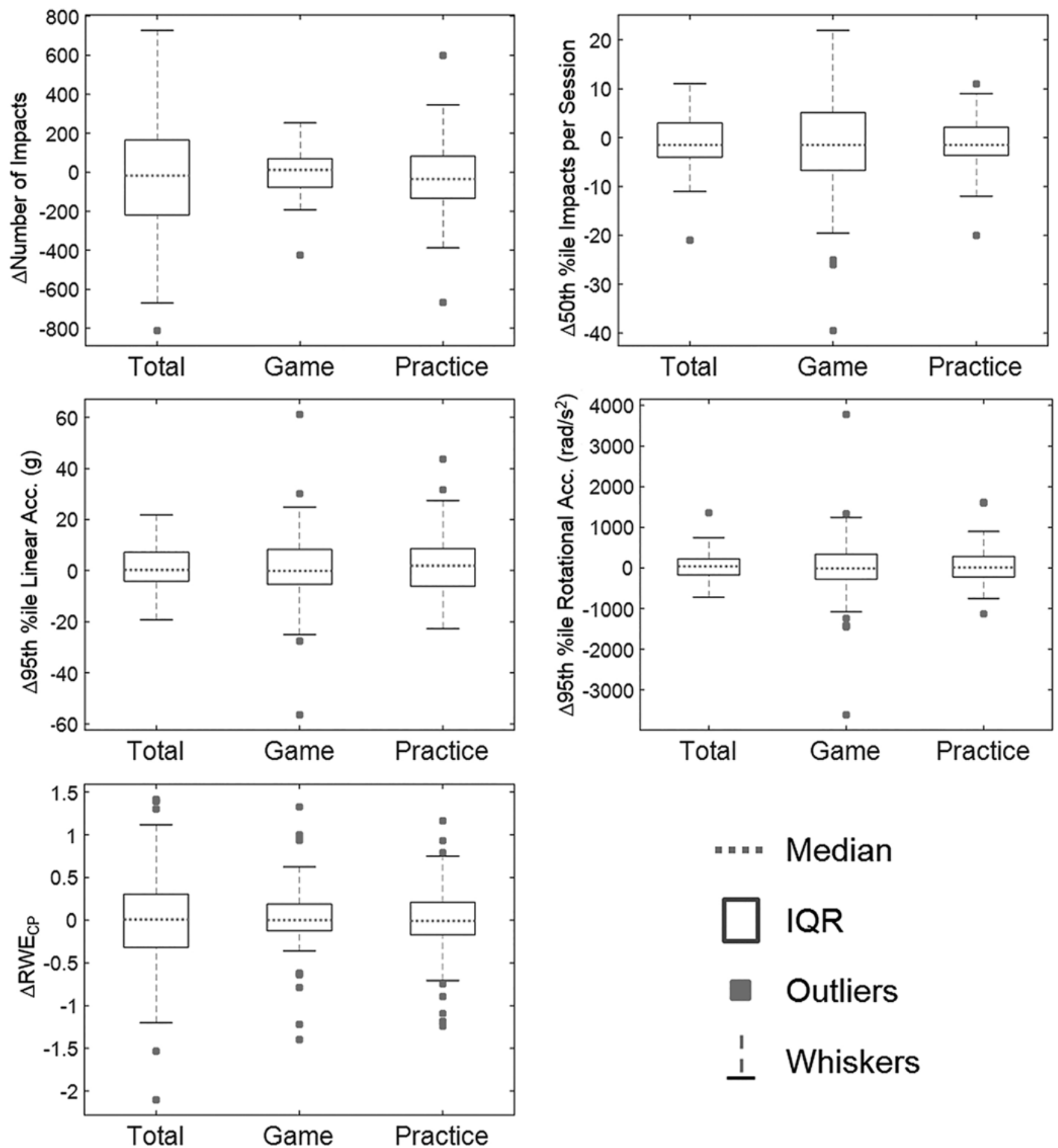

"*: Median
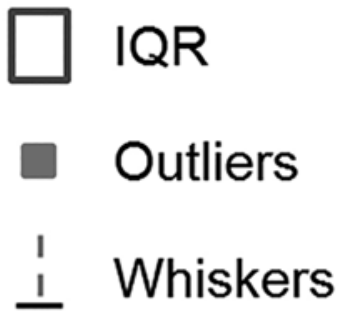

FIG. 2. Box plots summarizing changes in all HIE metrics for returning athletes over consecutive seasons of youth football. A positive change indicates an increase in $\mathrm{HIE}$ from one season to the next, and a negative change indicates a decrease in HIE from one season to the next. Whiskers include data within 1.5 times the interquartile range (IQR) from the lowest and highest quartile. Acc. $=$ acceleration; \%ile = percentile.

While general trends of increasing HIE with athlete's age and level of play in football, as well as variations in HIE among individuals, are evident in the literature, the results from this study demonstrate that changes in HIE between seasons vary considerably among individual ath- letes. ${ }^{1,3-5}$ There were trends of increasing mean number of game impacts, 50th percentile impacts per session, and 50th percentile impacts per game, but the differences were not significant. Therefore, there are likely not consistent increases in HIE from season to season among youth foot- 
TABLE 2. Summary of HIE metrics for each season

\begin{tabular}{|c|c|c|c|}
\hline & Season 1 & Season 2 & Season 3 \\
\hline \multicolumn{4}{|l|}{ Season } \\
\hline No. of impacts* & $317.9(236.4-427.5)$ & $296.0(230.9-379.4)$ & $322.3(187.9-553.0)$ \\
\hline 50th \%ile impacts/session & $7.1(5.3-9.5)$ & $7.4(5.8-9.4)$ & $8.0(4.7-13.5)$ \\
\hline 95th \%ile LA in $g$ & $52.9(49.7-56.3)$ & $51.1(48.5-53.9)$ & $47.9(42.7-53.9)$ \\
\hline 95th \%ile RA in rad $/ \mathrm{sec}^{2 *}$ & $2451.6(2307.7-2604.8)$ & $2412.0(2293.0-2536.9)$ & 2375.6 (2127.7-2652.1) \\
\hline $\mathrm{RWE}_{\mathrm{CP}}^{*}$ & $0.34(0.22-0.53)$ & $0.37(0.25-0.53)$ & $0.25(0.11-0.56)$ \\
\hline \multicolumn{4}{|l|}{ Games } \\
\hline No. of impacts* & $86.0(59.2-125.0)$ & $96.0(70.3-131.1)$ & 132.5 (67.2-261.5) \\
\hline 50th \%ile impacts/session & $9.6(6.7-13.7)$ & $9.7(7.1-13.1)$ & $10.2(5.3-19.7)$ \\
\hline 95th \%ile LA in $g$ & $54.2(49.9-58.7)$ & $53.5(49.8-57.5)$ & $50.1(43.3-57.9)$ \\
\hline 95th \%ile RA in rad/sec ${ }^{2}$ & 2495.9 (2291.4-2718.9) & $2567.0(2381.8-2766.7)$ & $2611.3(2239.7-3044.2)$ \\
\hline $\mathrm{RWE}_{\mathrm{CP}}$ & $0.08(0.04-0.14)$ & $0.12(0.07-0.20)$ & $0.12(0.04-0.37)$ \\
\hline \multicolumn{4}{|l|}{ Practices } \\
\hline No. of impacts* & 207.8 (153.2-281.9) & $184.0(142.6-237.6)$ & $186.7(107.1-325.4)$ \\
\hline 50th \%ile impacts/session* & $6.7(5.2-8.8)$ & $6.8(5.5-8.6)$ & $6.7(4.1-10.8)$ \\
\hline 95th \%ile LA in $g$ & $52.6(49.0-56.6)$ & $50.4(47.4-53.6)$ & $49.3(43.2-56.3)$ \\
\hline 95th \%ile RA in rad/ $/ \mathrm{sec}^{2 *}$ & $2432.1(2267.0-2609.2)$ & $2341.2(2205.5-2484.9)$ & 2344.7 (2062.3-2665.4) \\
\hline $\mathrm{RWE}_{\mathrm{CP}}^{*}$ & $0.19(0.13-0.29)$ & $0.17(0.12-0.25)$ & $0.14(0.06-0.30)$ \\
\hline
\end{tabular}

$\mathrm{LA}=$ linear acceleration; $\mathrm{RA}=$ rotational acceleration; \%ile = percentile.

Values are expressed as mean $(95 \% \mathrm{Cl})$.

* Significant variation in HIE metrics among teams.

ball athletes. Our results suggest that an athlete's specific team explains significant variations in HIE among individuals. Prior studies have also shown that teams, player position, practice drills, and amount of contact can influence HIE. $2,4,8-10,38,41,42$ Youth football athletes may have a primary position, but it is common for athletes at this level to play multiple positions throughout the season. It is also likely that their primary position changes from season to season until they begin to specialize at the high school and college levels. While evaluating the effect of position on
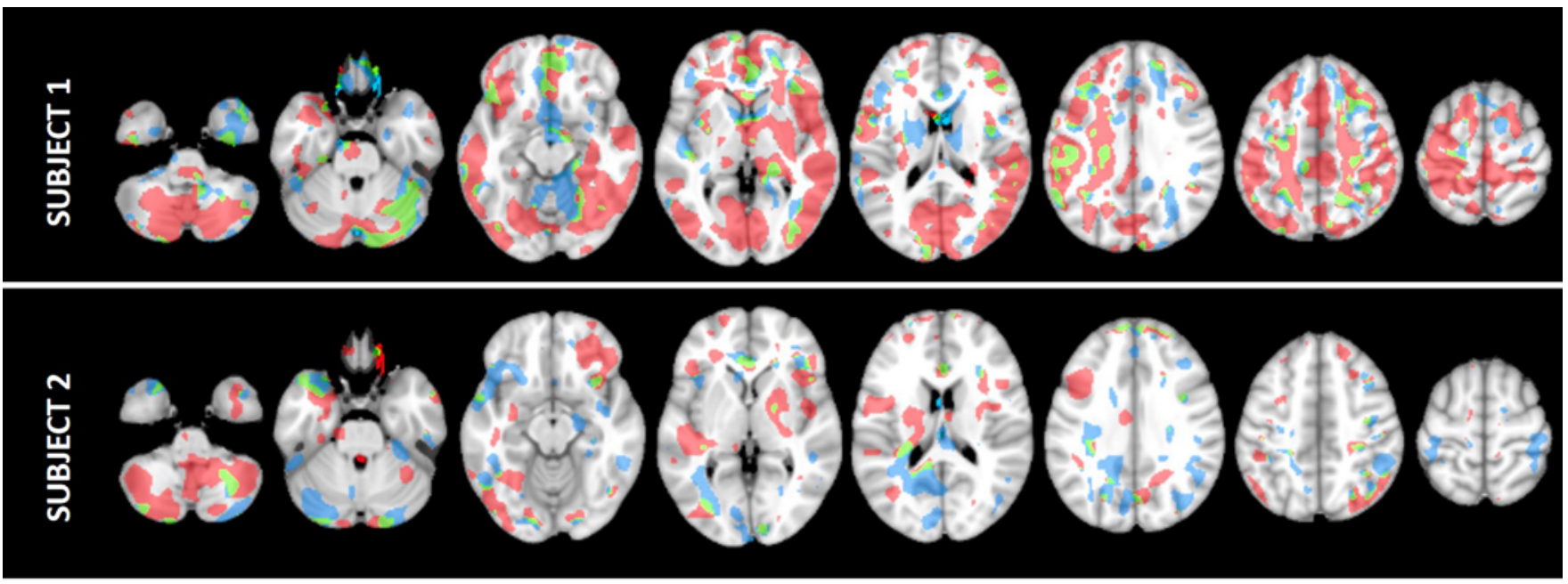

Abnormal voxels from season with $\underline{\text { high }} 50^{\text {th }}$ percentile impacts per practice

Abnormal voxels from season with low $50^{\text {th }}$ percentile impacts per practice

Overlapping abnormal voxels across both seasons

FIG. 3. Imaging data from two football athlete subjects showing a comparison between abnormal voxels from the season with the higher 50th percentile number of impacts per practice session and abnormal voxels from the season with the lower 50th percentile number of impacts per practice session. The season with the lower impact frequency in practice sessions was associated with fewer abnormal voxels compared to the season with a higher impact frequency in practice sessions for both athletes. 
TABLE 3. Summary of linear regression analysis results between $\triangle H I E$ metrics (predictor) and $\triangle D T I$ scalar metrics (outcome) controlling for average age of athletes and the difference in time between scans between seasons 1 and 2

\begin{tabular}{|c|c|c|c|c|c|c|c|c|c|c|}
\hline & \multicolumn{2}{|c|}{$\Delta \mathrm{FA}_{\text {voxels }}$} & \multicolumn{2}{|c|}{$\Delta \mathrm{MD}_{\text {voxels }}$} & \multicolumn{2}{|c|}{$\Delta \mathrm{C}_{\text {L-voxels }}$} & \multicolumn{2}{|c|}{$\Delta \mathrm{C}_{\mathrm{S} \text {-voxels }}$} & \multicolumn{2}{|c|}{$\Delta \mathrm{C}_{\mathrm{P} \text {-voxels }}$} \\
\hline & $p$ Value & Partial $\mathrm{R}^{2}$ & p Value & Partial $R^{2}$ & $p$ Value & Partial $R^{2}$ & $p$ Value & Partial $R^{2}$ & $p$ Value & Partial $R^{2}$ \\
\hline \multicolumn{11}{|l|}{ Season } \\
\hline$\Delta$ No. of impacts & 0.06 & 0.21 & 0.08 & 0.19 & 0.05 & 0.23 & 0.07 & 0.21 & 0.07 & 0.20 \\
\hline$\Delta 50$ th $\%$ ile impacts/session & 0.0004 & 0.58 & 0.3 & 0.07 & 0.0002 & 0.61 & 0.0002 & 0.62 & 0.002 & 0.49 \\
\hline$\Delta 95$ th $\%$ ile LA in $g$ & 0.6 & 0.02 & 0.7 & 0.01 & 0.4 & 0.04 & 0.6 & 0.02 & 0.7 & 0.01 \\
\hline$\Delta 95$ th $\%$ ile RA in rad $/ \mathrm{sec}^{2}$ & 0.7 & 0.01 & 0.9 & 0.00 & 0.7 & 0.01 & 0.7 & 0.01 & 0.8 & 0.00 \\
\hline$\Delta \mathrm{RWE}_{\mathrm{cp}}$ & 0.6 & 0.02 & 0.6 & 0.01 & 0.5 & 0.03 & 0.6 & 0.02 & 0.7 & 0.01 \\
\hline \multicolumn{11}{|l|}{ Games } \\
\hline$\Delta$ No. of impacts & 0.6 & 0.02 & 0.9 & 0.00 & 0.6 & 0.02 & 0.6 & 0.02 & 0.5 & 0.03 \\
\hline$\Delta 50$ th $\%$ ile impacts/session & 0.4 & 0.05 & 1.0 & 0.00 & 0.3 & 0.08 & 0.3 & 0.08 & 0.3 & 0.08 \\
\hline$\Delta 95$ th \%ile LA in $g$ & 0.7 & 0.01 & 0.9 & 0.00 & 0.6 & 0.02 & 0.6 & 0.02 & 0.6 & 0.02 \\
\hline$\Delta 95$ th $\%$ ile RA in rad $/ \mathrm{sec}^{2}$ & 1.0 & 0.00 & 1.0 & 0.00 & 0.8 & 0.01 & 0.8 & 0.00 & 0.9 & 0.00 \\
\hline$\Delta \mathrm{RWE}_{\mathrm{cp}}$ & 0.8 & 0.00 & 0.9 & 0.00 & 1.0 & 0.00 & 1.0 & 0.00 & 1.0 & 0.00 \\
\hline \multicolumn{11}{|l|}{ Practices } \\
\hline$\Delta$ No. of impacts & 0.02 & 0.32 & 0.01 & 0.34 & 0.01 & 0.34 & 0.02 & 0.30 & 0.03 & 0.28 \\
\hline$\Delta 50$ th $\%$ ile impacts/session & $<0.0001$ & 0.66 & 0.1 & 0.14 & $<0.0001$ & 0.67 & $<0.0001$ & 0.70 & 0.0008 & 0.54 \\
\hline$\Delta 95$ th $\%$ ile LA in $g$ & 0.2 & 0.13 & 0.4 & 0.04 & 0.08 & 0.19 & 0.2 & 0.12 & 0.2 & 0.11 \\
\hline$\Delta 95$ th $\%$ ile RA in rad $/ \mathrm{sec}^{2}$ & 0.9 & 0.00 & 0.8 & 0.00 & 0.8 & 0.01 & 0.8 & 0.00 & 1.0 & 0.00 \\
\hline$\Delta \mathrm{RWE}_{\mathrm{cp}}$ & 0.6 & 0.02 & 0.6 & 0.02 & 0.4 & 0.04 & 0.5 & 0.04 & 0.6 & 0.02 \\
\hline
\end{tabular}

Type III $p$ values and partial $R^{2}$ values are reported. Boldface type indicates statistical significance at $p<0.05$.

HIE over multiple seasons was beyond the scope of this study, it should be considered in future studies of youth football athletes. ${ }^{10,41}$ As youth athletes change teams and move up the levels from season to season, their coaches, teammates, practice structure, and types of drills change too, all of which may influence their HIE.

There were several significant positive correlations between changes in the total number of impacts and impacts per session, especially during practices, and changes in neuroimaging metrics. These results suggest that, among individual athletes, increases in HIE from one season to the next were associated with a greater number of abnormal voxels and that decreases in HIE were associated with fewer numbers of abnormal voxels from one season to the next (Fig. 4). There are a growing number of studies showing significant associations between HIE metrics and both neuroimaging and cognitive function during single-season evaluations of youth through collegiate football players, even in the absence of a clinically diagnosed concussion..$^{22,25,29,35,43,44}$ For example, a study of 80 collegiate football and ice hockey players found that several measures of HIE, including the number of impacts and the 95th percentile linear and rotational accelerations, were significantly correlated with several DTI scalar metrics in various brain regions over the course of a single season..$^{29}$ The nature of this relationship is not yet fully understood, and there are conflicting results that may be due to variations in methodology. ${ }^{35,44}$ While there is evidence of structural changes
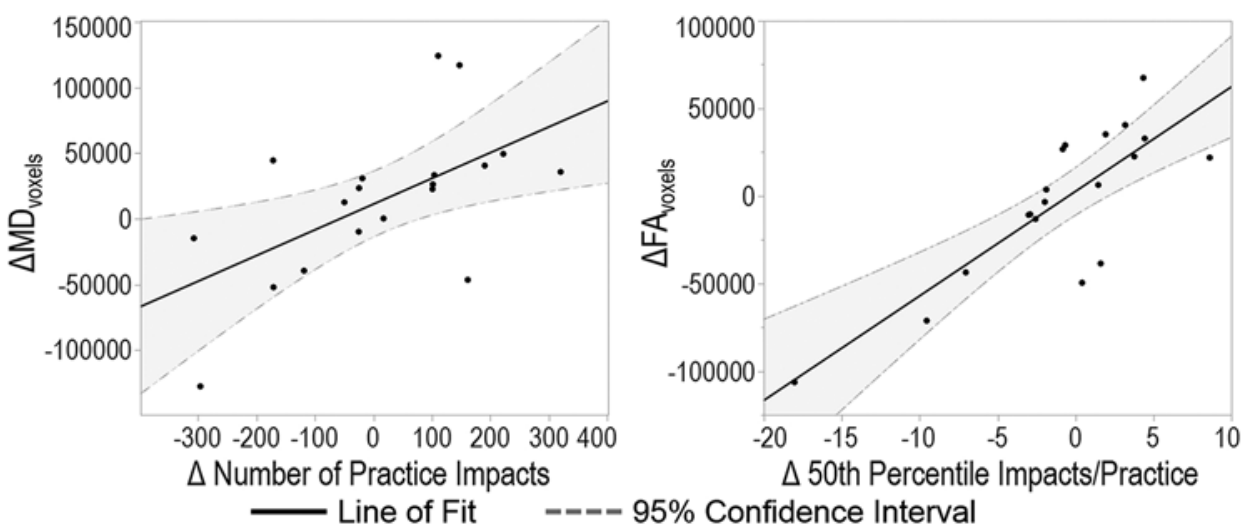

FIG. 4. Examples of the significant correlations reported in Table 3. Left: $\Delta$ number of practice impacts versus $\Delta \mathrm{MD}_{\text {voxels }}$. Right: $\Delta 50$ th percentile impacts per practice versus $\Delta \mathrm{FA}_{\text {voxels }}$. 
in the brain that are positively correlated with HIE during a season of football, the number of abnormal DTI voxels may remain the same or even decrease from one season to the next. Our study did not address fluctuation in abnormal voxels from the end of one season to the beginning of the next, because we were unable to adequately monitor offseason activities. Regardless, these results provide further evidence that HIE, especially the number and frequency of impacts, may be a modulating factor in DTI changes over multiple seasons of contact sports.

This study expands our understanding of longitudinal changes in neuroimaging and how they relate to subconcussive HIE. Prior longitudinal studies have sought to determine whether changes in white matter integrity remain after exposure to subconcussive head impacts ends. One study found that white matter changes persisted after 6 months of no-contact rest, and another study found that white matter changes returned to baseline. ${ }^{23,36}$ Yet, no prior study has evaluated how changes in HIE may influence DTI metrics over multiple seasons. By demonstrating a significant association between the total number of practice impacts and impacts per session, overall and in practice, with DTI scalar metrics, this study contributes to a growing body of evidence that subconcussive head impacts may influence brain changes. Additionally, it is important to note that we observed reductions in the number of abnormal voxels from season to season in some individuals, which may relate to reduced HIE. The results of this study, in conjunction with those of prior work, prompt future longitudinal investigations into white matter integrity over multiple years of exposure to repetitive head impacts, as well as during periods of no-contact rest among youth football athletes. A challenge with longitudinal studies of youth athletes, however, is that the athletes often participate in several sports, including other contact sports, which must be considered when assessing an athlete's cumulative HIE. Continued longitudinal data collection of neuroimaging and HIE data are needed to understand how changes in DTI scalar metrics over multiple years relate to long-term clinical and functional outcomes.

Changes in the number of practice impacts, 50th percentile impacts per practice, and 50th percentile impacts per session were all significantly correlated with $\Delta$ DTI scalar metrics; however, changes in the 95th percentile linear and rotational head accelerations and $\mathrm{RWE}_{\mathrm{CP}}$ were not associated with changes in neuroimaging from one season to the next. Head impact magnitude and cumulative exposure are still important factors to a football player's HIE, especially as they relate to concussion risk, and they have been associated with single-season changes in neuroimaging metrics. ${ }^{22,25,43}$ It was outside the scope of this study to evaluate the reason for the lack of this relationship. However, it may be due to several factors, such as the minimal variation in 95th percentile head acceleration and $R W E_{C P}$ values among this cohort, especially compared to variations found in high school athletes who have more defined playing positions and, therefore, more distinct differences in HIE among players. Additionally, changes in game-specific HIE metrics were not associated with changes in neuroimaging findings. Changes in the number of abnormal voxels in this cohort were closely associated with changes in the number and frequency of impacts, especially during practice sessions, from one season to the next. The significant associations with changes in HIE in practices and overall, but not in games, may be due to the fact that the majority of impacts occur in practice for most athletes, and practice sessions occur more frequently than games. ${ }^{1,4,38}$ Furthermore, HIE, and in particular, the number and frequency of head impacts, varies considerably depending on drills and practice structure, which demonstrates the influence that coaches and practice structure have on HIE. 2,9,42 For instance, one high school team limited contact to no more than two collision practice days in a week, which resulted in an average $42 \%$ reduction in the total number of head impacts. ${ }^{42}$ Reducing the total number of impacts and the number of impacts in each session, especially in practice, may limit increases in abnormal imaging findings over multiple seasons of participation in football.

\section{Limitations}

This study has several limitations that must be considered. While this is the only study to date that includes longitudinal biomechanical and imaging data sets of youth football players, the sample size is still relatively small, and all study participants were from the same region and functioned under the same national youth football organization. Additionally, other confounding factors may have influenced the results, such as participation in other contact sports before or during the multiple years the athletes participated in the study. The focus of this study was primarily the longitudinal biomechanical head impact data, and, while a subsample of participants with biomechanics data had complete and usable imaging data for multiple seasons, there were several factors that limited collecting complete imaging data for all 47 athletes over multiple seasons. These limiting factors included failure to keep imaging appointments on the part of athlete participants or parents/guardians, motion artifacts, clinical abnormalities, and budgetary constraints. While the longitudinal imaging data sets were limited in sample size, this study and associated data may serve as a comparison to, or a foundation for, future targeted longitudinal studies. The selected threshold to identify abnormally high or low voxel-wise z-scores (2 SDs) was chosen based on previous experience and was used to facilitate comparisons with previous mild traumatic brain injury studies, which have also included this threshold. ${ }^{24,25,35}$ We also conducted a sensitivity analysis, repeating the regression analyses by using a range of SD z-score map cutoffs (1.0-3.0), which demonstrated the findings to be robust, and, while increasing the SD cutoff attenuates some associations, the general trends and conclusions still hold. A group of 16 noncontact sport control athletes were used to create a normative reference for each scalar metric and to compute voxel-wise z-scores; however, longitudinal imaging data were not part of the control athlete cohort, and control athletes were only scanned at pre- and postseasonal time points for a single season. Future studies should aim to include longitudinal imaging data sets from noncontact sport control athletes. Changes in cognitive function were not evaluated in this study, so the functional and clinical implications of the changes in neuroimaging over two consecutive seasons seen in this 
study are not yet known. Last, it is possible that some head impacts were not measured due to equipment battery malfunctions or other hardware issues, but trained research staff attended every session to monitor data collection and to promptly resolve any issues that arose. There is also some measurement error associated with the HIT System. However, the error associated with linear and rotational acceleration measurements is within the range of acceptable error for other measurement devices. ${ }^{45}$

\section{Conclusions}

The results from this longitudinal study demonstrate that HIE can increase or decrease between seasons in individual youth football athletes and that there were no significant changes in HIE from one season to the next in this cohort. There were both increases and decreases in the number of abnormal DTI voxels from season 1 to season 2 , and those changes were significantly positively correlated with changes in the number of practice impacts, 50th percentile number of impacts per practice, and 50th percentile number of impacts per session. Although the data from prior studies have shown single-season associations between HIE metrics and neuroimaging measures, the findings of this study suggest that a reduction in the number and frequency of head impacts during practice sessions may reduce the number of abnormal imaging findings from one season to the next in youth football. These results support continued efforts to reduce HIE in practice. Additionally, future investigations into the longterm relationship between HIE and neuroimaging findings are warranted to better understand how reduction in subconcussive HIE may affect the brain over many years of participation in football.

\section{Acknowledgments}

Research reported in this publication was supported by the National Institute of Neurological Disorders and Stroke of the National Institutes of Health under award numbers R01NS094410 and R01NS082453. The National Center for Advancing Translational Sciences, National Institutes of Health, supported Dr. Urban through grant KL2TR001421. The content is solely the responsibility of the authors and does not necessarily represent the official views of the National Institutes of Health. We give special thanks to the Childress Institute for Pediatric Trauma at Wake Forest Baptist Medical Center for providing support for this study. We thank the youth football organization's board members, coaches, parents, and athletes whose support makes this research possible. We thank the athletic trainers for their support. We also thank all the research assistants who helped with data collection.

\section{References}

1. Broglio SP, Surma T, Ashton-Miller JA. High school and collegiate football athlete concussions: a biomechanical review. Ann Biomed Eng. 2012;40(1):37-46.

2. Cobb BR, Urban JE, Davenport EM, et al. Head impact exposure in youth football: elementary school ages 9-12 years and the effect of practice structure. Ann Biomed Eng. 2013;41(12): 2463-2473.

3. Crisco JJ, Wilcox BJ, Beckwith JG, et al. Head impact exposure in collegiate football players. J Biomech. 2011;44(15): 2673-2678.

4. Kelley ME, Urban JE, Miller LE, et al. Head impact expo- sure in youth football: comparing age- and weight-based levels of play. J Neurotrauma. 2017;34(11):1939-1947.

5. Urban JE, Davenport EM, Golman AJ, et al. Head impact exposure in youth football: high school ages 14 to 18 years and cumulative impact analysis. Ann Biomed Eng. 2013;41(12): 2474-2487.

6. Kontos AP, Elbin RJ, Fazio-Sumrock VC, et al. Incidence of sports-related concussion among youth football players aged 8-12 years. J Pediatr. 2013;163(3):717-720.

7. Dompier TP, Kerr ZY, Marshall SW, et al. Incidence of concussion during practice and games in youth, high school, and collegiate American football players. JAMA Pediatr. 2015; 169(7):659-665.

8. Kelley ME, Kane JM, Espeland MA, et al. Head impact exposure measured in a single youth football team during practice drills. J Neurosurg Pediatr. 2017;20(5):489-497.

9. Asken BM, Brooke ZS, Stevens TC, et al. Drill-specific head impacts in collegiate football practice: implications for reducing "friendly fire" exposure. Ann Biomed Eng. 2019; 47(10):2094-2108.

10. Mihalik JP, Bell DR, Marshall SW, Guskiewicz KM. Measurement of head impacts in collegiate football players: an investigation of positional and event-type differences. Neurosurgery. 2007;61(6):1229-1235.

11. Montenigro PH, Alosco ML, Martin BM, et al. Cumulative head impact exposure predicts later-life depression, apathy, executive dysfunction, and cognitive impairment in former high school and college football players. J Neurotrauma. 2017;34(2):328-340.

12. Young TJ, Daniel RW, Rowson S, Duma SM. Head impact exposure in youth football: elementary school ages 7-8 years and the effect of returning players. Clin J Sport Med. 2014; 24(5):416-421.

13. McKee AC, Alosco ML, Huber BR. Repetitive head impacts and chronic traumatic encephalopathy. Neurosurg Clin N Am. 2016;27(4):529-535.

14. McKee AC, Cantu RC, Nowinski CJ, et al. Chronic traumatic encephalopathy in athletes: progressive tauopathy after repetitive head injury. J Neuropathol Exp Neurol. 2009;68(7): 709-735.

15. Broglio SP, Eckner JT, Paulson HL, Kutcher JS. Cognitive decline and aging: the role of concussive and subconcussive impacts. Exerc Sport Sci Rev. 2012;40(3):138-144.

16. Mez J, Daneshvar DH, Kiernan PT, et al. Clinicopathological evaluation of chronic traumatic encephalopathy in players of American football. JAMA. 2017;318(4):360-370.

17. Stamm JM, Bourlas AP, Baugh CM, et al. Age of first exposure to football and later-life cognitive impairment in former NFL players. Neurology. 2015;84(11):1114-1120.

18. Solomon GS, Kuhn AW, Zuckerman SL, et al. Participation in pre-high school football and neurological, neuroradiological, and neuropsychological findings in later life: a study of 45 retired National Football League players. Am J Sports Med. 2016;44(5):1106-1115.

19. Baugh CM, Stamm JM, Riley DO, et al. Chronic traumatic encephalopathy: neurodegeneration following repetitive concussive and subconcussive brain trauma. Brain Imaging Behav. 2012;6(2):244-254.

20. McKee AC, Stern RA, Nowinski CJ, et al. The spectrum of disease in chronic traumatic encephalopathy. Brain. 2013; 136(Pt 1):43-64.

21. Hazrati LN, Tartaglia MC, Diamandis P, et al. Absence of chronic traumatic encephalopathy in retired football players with multiple concussions and neurological symptomatology. Front Hum Neurosci. 2013;7:222.

22. Bahrami N, Sharma D, Rosenthal S, et al. Subconcussive head impact exposure and white matter tract changes over a single season of youth football. Radiology. 2016;281(3): 919-926. 
23. Bazarian JJ, Zhu T, Zhong J, et al. Persistent, long-term cerebral white matter changes after sports-related repetitive head impacts. PLoS One. 2014;9(4):e94734.

24. Davenport EM, Apkarian K, Whitlow CT, et al. Abnormalities in diffusional kurtosis metrics related to head impact exposure in a season of high school varsity football. J Neurotrauma. 2016;33(23):2133-2146.

25. Davenport EM, Whitlow CT, Urban JE, et al. Abnormal white matter integrity related to head impact exposure in a season of high school varsity football. J Neurotrauma. 2014; 31(19):1617-1624.

26. Giza CC, Hovda DA. The new neurometabolic cascade of concussion. Neurosurgery. 2014;75(0 4):S24-S33.

27. Bazarian JJ, Zhong J, Blyth B, et al. Diffusion tensor imaging detects clinically important axonal damage after mild traumatic brain injury: a pilot study. J Neurotrauma. 2007;24(9): 1447-1459.

28. Mayer AR, Ling J, Mannell MV, et al. A prospective diffusion tensor imaging study in mild traumatic brain injury. Neurology. 2010;74(8):643-650.

29. McAllister TW, Ford JC, Flashman LA, et al. Effect of head impacts on diffusivity measures in a cohort of collegiate contact sport athletes. Neurology. 2014;82(1):63-69.

30. Hulvershorn LA, Cullen K, Anand A. Toward dysfunctional connectivity: a review of neuroimaging findings in pediatric major depressive disorder. Brain Imaging Behav. 2011;5(4): 307-328.

31. Rane P, Cochran D, Hodge SM, et al. Connectivity in autism: a review of MRI connectivity studies. Harv Rev Psychiatry. 2015;23(4):223-244.

32. Messina R, Rocca MA, Colombo B, et al. White matter microstructure abnormalities in pediatric migraine patients. Cephalalgia. 2015;35(14):1278-1286.

33. Oishi K, Mielke MM, Albert M, et al. DTI analyses and clinical applications in Alzheimer's disease. J Alzheimers Dis. 2011;26(suppl 3):287-296.

34. Guskiewicz KM, Valovich McLeod TC. Pediatric sportsrelated concussion. PM R. 2011;3(4):353-364.

35. Davenport EM, Urban JE, Mokhtari F, et al. Subconcussive impacts and imaging findings over a season of contact sports. Concussion. 2016;1(4):CNC19.

36. Mayinger MC, Merchant-Borna K, Hufschmidt J, et al. White matter alterations in college football players: a longitudinal diffusion tensor imaging study. Brain Imaging Behav. 2018; 12(1):44-53.

37. Yuan W, Barber Foss KD, Thomas S, et al. White matter alterations over the course of two consecutive high-school football seasons and the effect of a jugular compression collar: a preliminary longitudinal diffusion tensor imaging study. Hum Brain Mapp. 2018;39(1):491-508.

38. Kelley ME, Espeland MA, Flood WC, et al. Comparison of head impact exposure in practice drills among multiple youth football teams. J Neurosurg Pediatr. 2018;23(3):381-389.

39. Crisco JJ, Chu JJ, Greenwald RM. An algorithm for estimating acceleration magnitude and impact location using multiple nonorthogonal single-axis accelerometers. J Biomech Eng. 2004;126(6):849-854.
40. Rowson S, Duma SM. Brain injury prediction: assessing the combined probability of concussion using linear and rotational head acceleration. Ann Biomed Eng. 2013;41(5):873-882.

41. Broglio SP, Sosnoff JJ, Shin S, et al. Head impacts during high school football: a biomechanical assessment. $J$ Athl Train. 2009;44(4):342-349.

42. Broglio SP, Williams RM, O’Connor KL, Goldstick J. Football players' head-impact exposure after limiting of fullcontact practices. J Athl Train. 2016;51(7):511-518.

43. Talavage TM, Nauman EA, Breedlove EL, et al. Functionally-detected cognitive impairment in high school football players without clinically-diagnosed concussion. $\mathrm{J} \mathrm{Neu}$ rotrauma. 2014;31(4):327-338.

44. Slobounov SM, Walter A, Breiter HC, et al. The effect of repetitive subconcussive collisions on brain integrity in collegiate football players over a single football season: a multi-modal neuroimaging study. Neuroimage Clin. 2017;14: 708-718.

45. Beckwith JG, Greenwald RM, Chu JJ. Measuring head kinematics in football: correlation between the Head Impact Telemetry System and Hybrid III headform. Ann Biomed Eng. 2012;40(1):237-248.

\section{Disclosures}

The authors report no conflict of interest concerning the materials or methods used in this study or the findings specified in this paper.

\section{Author Contributions}

Conception and design: Urban, Kelley, Davenport, Stitzel. Acquisition of data: Urban, Kelley, Jones, Davenport, Miller. Analysis and interpretation of data: Urban, Kelley, Davenport. Drafting the article: Urban, Kelley, Davenport, Maldjian, Stitzel. Critically revising the article: Urban, Kelley, Jones, Davenport, Whitlow, Maldjian, Stitzel. Reviewed submitted version of manuscript: all authors. Statistical analysis: Kelley, Snively. Administrative/technical/material support: Urban, Powers, Whitlow, Maldjian, Stitzel. Study supervision: Urban, Whitlow, Maldjian, Stitzel.

\section{Supplemental Information \\ Previous Presentations}

A portion of this work was presented at the 2019 Summer Biomechanics, Bioengineering, and Biotransport Conference, Seven Springs, PA, June 25-28, 2019.

\section{Correspondence}

Jillian E. Urban: Virginia Tech-Wake Forest School of Biomedical Engineering and Sciences, Winston-Salem, NC. jurban@ wakehealth.edu. 